Economía, Sociedad y Territorio, vol. vIII, núm. 28, 2008, 965-980

\title{
Virtudes del recuerdo: un acercamiento a las memorias colectivas en México
}

\section{The virtues of remembrance: a close-up into the collective memories in Mexico}

SALVAdor SigÜEnZa-Orozco*

\begin{abstract}
In this paper we consider memory as a collective process with social and cultural implications. We analyse the collective memories in Mexico by classifying them into two categories: traditional and civic. The former is attributed to indigenous people and the latter is spread and reproduced by the construction process of the national state. Although it is true that this separation can be somewhat arbitrary, in every-day life they are linked together and show certain equivalences as they are cultural processes that make use of different mechanisms for their reproduction. We have taken into account the experiences of indigenous leaders and authorities that have pondered the importance of their local ways of organisation. We consider that it is important to improve our knowledge of these processes in order to contribute to the respect of both the processes themselves and the people that assume them.
\end{abstract}

Keywords: collective memory, traditional memory, civic memory, education, indigenous people.

\section{Resumen}

En este trabajo se considera a la memoria como un proceso colectivo con implicaciones sociales y culturales. Se analizan las memorias colectivas en México caracterizándolas en dos tipos: tradicional y cívica. La primera se atribuye a los pueblos indígenas, la segunda promovida y reproducida por el Estado nacional en su proceso de construcción. Y si bien para su comprensión se separan arbitrariamente, en la vida cotidiana suelen encontrarse articuladas y presentan equivalencias en tanto procesos culturales que recurren a diferentes mecanismos para reproducirse. Se tomaron en cuenta las experiencias de líderes y autoridades indígenas que han reflexionado acerca de la importancia de sus formas de organización local. Se considera importante conocer mejor estos procesos para contribuir al respeto de los mismos y de la gente que los asume.

Palabras clave: memoria colectiva, memoria tradicional, memoria cívica, educación, pueblos indígenas.

* CIESAS, Unidad Pacífico Sur (antes Oaxaca). Correo-e: salvadorsgz@yahoo.com. 


\section{Introducción}

A partir de los años veinte del siglo pasado empezaron a llegar a varias regiones del país, muchas de ella habitadas por pueblos indígenas, los maestros misioneros de la Secretaría de Educación Pública. La labor de estos profesores apuntaba a enseñar formas superiores de cultura a gente que aún vivía "como lo hacían las tribus indígenas en tiempos de la conquista" (AHSEP, caja 171, exp. 1). Las actividades docentes requerían necesariamente del proceso de castellanización ya que la inmensa mayoría de la población era monolingüe indígena. El proceso educativo que se presentaba tenía su grado de complejidad ya que implicaba modificar por completo formas y hábitos de tradición milenaria que se confrontaban con los valores de la educación nacional, la cual promovía -entre otros temas- la enseñanza de un pasado nacional común, forjador de la identidad mexicana. Este pasado, esta memoria de la cual el magisterio era portavoz, propuso insertarse en una población con una memoria colectiva previa.

\section{La memoria}

La memoria es registro y reconstrucción, es un proceso que se comunica (memoria comunicada) y se hereda (memoria heredada) de forma cultural y social. Al ser colectiva se erige en un conjunto de significados compartido y asumido por un grupo de personas, a través de ella dicha comunidad se construye y existe. Maurice Halbwachs afirma que sólo hay una memoria, resultado de la articulación social, la cual tiene marcos sociales que se pueden dividir en generales (espacio, tiempo, lenguaje) y específicos (relativo a diferentes grupos sociales). Estos referentes, estos marcadores, permiten el establecimiento de un sistema global de pasado a través del cual se puede rememorizar de manera individual y colectiva (Halbwachs, 2004). Lo anterior se puede explicar mejor si consideramos que los recuerdos, por muy personales que sean, existen vinculados con la vida de la sociedad de la que se es miembro.

De los marcos sociales señalados por Halbwachs, el lenguaje es el sistema mediatizador que transmite la experiencia y el pensamiento a los demás; más aún, la transmisión de la experiencia y el pensamiento específicos requieren un lenguaje determinado (Vigotsky, 1996). La función fundamental de las palabras es la comunicación, es decir, el contacto social. Sin embargo, se debe 
considerar que no es lo mismo el lenguaje oral que el escrito, ya que mientras aquél no permite procesos de formulación complicada y su velocidad no da tiempo a deliberar y elegir con claridad, en el lenguaje escrito la comunicación se logra por medio de palabras complicadas y se erige en la forma más elaborada de lenguaje. Una consideración adicional acerca del lenguaje es que, como marco social, es el más elemental y estable para la memoria, tanto que podría afirmarse que en general ésta depende de él. Por ello la verdadera comunicación presupone una actitud generalizadora, lo cual implica la unificación lingüística (pensamientos, códigos) ya que es difícil definir un concepto fuera de su contexto.

A partir de la creación del Estado nacional y, sobre todo, en su paulatino proceso de consolidación, éste se asumió como responsable de la memoria colectiva y como portador de la misma, integrándola mediante la selección de viejas memorias e incluyendo nuevos contenidos, para posteriormente reinterpretarla y difundirla. Este fue uno de los mecanismos para construir la identidad nacional.

En el caso de México, el Estado que surge después de la Revolución iniciada en 1910 asumió que era fundamental establecer un nuevo régimen basado en el principio republicano de división de poderes y en la atención de demandas sociales de la población. Para lograrlo, se recurrió a diversos mecanismos entre los que la educación tuvo un papel esencial, pues se le asignó el llevar a los rincones de la patria las buenas nuevas del régimen revolucionario. Así, la escuela y los maestros participaron de manera significativa en el naciente esfuerzo de ingeniería social para atender los rezagos de la población, tarea que en muchas regiones del país tuvo fuerte impacto en las cinco décadas posrevolucionarias, años en los que la escuela prácticamente fue la única institución oficial que arribó a ellas.

Pero, en muchos lugares del país, la memoria que la escuela mexicana empezó a introducir y reproducir de manera sistemática, se encontró con formas de organización y tradiciones locales fuertemente arraigadas, muchas de ellas con prácticas construidas a partir de elementos de las culturas prehispánicas y retomando criterios de la administración pública implantados en la época colonial.

Con base en este planteamiento considero la existencia de dos tipos de memoria que llamaré memoria tradicional y memoria cívica. Ambos son fenómenos de carácter individual y social 
que contienen elementos conscientes e inconscientes en la interpretación y renovación del pasado; asimismo los dos son procesos culturales que recurren a diferentes mecanismos y tienen determinadas características y contenidos. Sólo que identifico a la memoria cívica como resultado del colosal esfuerzo del Estado-nación por crear su memoria; es decir, una memoria de carácter institucional, estatalizada. Por otra parte, la memoria tradicional es la de los pueblos indígenas, una memoria amplia y no institucional que tiene fuerte arraigo si se considera la tradición como algo arraigado en el pasado.

Durante gran parte del siglo XIX y en el Xx en México hubo un debate público, cada vez más amplio, sobre lo que se consideraba culto y lo que se juzgaba popular, entre lo que se valoraba moderno y lo apreciado como tradicional; así como los medios adecuados para transmitirse y reproducirse (o no), entre los que la escuela tuvo un papel principal. Asocio, entonces, lo culto-moderno a la memoria cívica y lo popular-tradicional a la memoria tradicional; aunque lo cierto es que ambos tipos de memoria se hibridaron recuperando contenidos mutuamente. Más aún, frente a la memoria cívica la tradicional se conservó -en ocasiones de manera clandestina- y posteriormente emergió para reclamar su espacio; un lugar que, en ocasiones, realmente no había abandonado.

\section{Memoria tradicional y memoria cívica}

En el afán por comprender mejor la diferencia que planteo entre ambos tipos de memoria, aislaré deliberadamente cada una de ellas para examinar los elementos que las componen, no porque estén separados sino para contribuir a su mejor comprensión, sobre todo de la tradición indígena.

Considero que la memoria tradicional ${ }^{1}$ es el conjunto de valores, normas, rasgos culturales, formas de creencia, así como usos y costumbres presentes en las prácticas y en los procesos comunitarios de los pueblos indígenas. Estos pueblos cuentan con una personalidad colectiva, una identidad comunitaria que se mani-

\footnotetext{
${ }^{1}$ Este concepto lo elaboré a partir de mi experiencia familiar y personal, así como mi formación académica. Familiar porque desciendo de indígenas mixes, quienes se han desempeñado como principales en el pueblo de San Pedro y San Pablo Ayutla. La experiencia personal se refiere al trabajo de campo con indígenas zapotecos durante la realización de la tesis de licenciatura (1990-1992), así como el trato con diferentes pueblos indígenas de Oaxaca durante los años que colaboré en el Instituto Oaxaqueño de las Culturas (1993-1999). El concepto se enriqueció durante conversaciones con investigadores del CIESAS Pacífico Sur y del INAH Oaxaca.
} 
fiesta en su lengua, su cosmogonía, sus tradiciones, sus costumbres y sus formas de organización. Lo anterior implica la transmisión de valores grupales, la visión sacralizada de la realidad, el respeto a las autoridades comunales y el uso (en ocasiones exclusivo) de la lengua indígena. La memoria tradicional se comunica a través de sistemas orales de producción del conocimiento, el cual se acumula y transmite de manera intergeneracional. Al ser de carácter oral, colectivo e histórico; no requiere de una institución oficial para aprenderse, se asimila con la lengua y las prácticas habituales de la vida misma -la familia, la comunidadque la afianzan cotidianamente ya que, como afirma Pitarch, es una categoría de experiencia (2002).

Por su parte, la memoria cívica ${ }^{2}$ está compuesta por los rasgos transmitidos oficialmente y que identifican al mexicano, como la historia y la lengua nacionales; generalmente tiene un soporte escrito (el libro y en especial el libro escolar de texto gratuito), se comunica a través de instituciones (como la escuela) y se apoya en funcionarios (como los maestros), lo que permite su repetición, expansión y afianzamiento. ${ }^{3}$ Esta memoria es parte de una política de Estado, es decir, los contenidos educativos son parte de una política pública oficial que socializa y nacionaliza de manera hegemónica, entendiendo con Gramsci que el Estado no es sólo un control burocrático sino un todo que comprende la cultura de un pueblo determinado. Al construirse una concepción hegemónica se acepta la construcción del poder preeminente de un grupo, cuyos criterios y valores generalmente se aceptan (Gramsci, 1975).

La memoria cívica tiene un carácter predominantemente político y social, busca homogeneizar las conciencias a través de instituciones de socialización con el fin de afirmar los componentes territoriales, políticos y culturales de la nación. La cultura pública, en especial el sistema educativo (generalizado, público y unificado), funciona como mecanismo de cohesión social para que la nación subsista de una generación a otra.

\footnotetext{
${ }^{2}$ Para explicar lo que entiendo por memoria cívica tomo como punto de partida mi formación como profesor y mi experiencia en la Secretaría de Educación Pública (SEP). Además, el concepto fue ampliamente discutido con Antonio Niño durante la elaboración de mi tesis doctoral, de la cual fue director.

${ }^{3}$ En la escuela mexicana, particularmente en la primaria, la enseñanza de las ciencias sociales -mucho tiempo dirigida de manera enfática a la instrucción de la historia y a la celebración de los festejos nacionales-, contribuyó a que la historia y el civismo se erigieran en el soporte del deber ser del mexicano; dando así sustento a esta memoria que he llamado cívica.
} 
Los elementos de ambas memorias no están desarticulados, se vinculan y relacionan mediante un intercambio con base en intereses y perspectivas locales, que pueden ser motivadas por el entorno físico o humano. La memoria tradicional se manifiesta en una sociedad de actores colectivos, miembros de una comunidad con valores de grupo; la memoria cívica se produce a partir de individuos legalmente iguales y teóricamente homogéneos que participan en un proceso de socialización basado en el sistema educativo oficial. La hegemonía con la que se puede caracterizar a la memoria cívica provoca que, al mismo tiempo que hay dominio también hay resistencia de la memoria tradicional, sobre todo porque el control y la subordinación no son sinónimo de lealtad genuina.

Una primera caracterización de ambos tipos de memoria se puede apreciar en el siguiente cuadro.

\begin{tabular}{ll}
\hline \multicolumn{1}{c}{ Memoria tradicional } & \multicolumn{1}{c}{ Memoria cívica } \\
\hline Identidad comunitaria & Identidad individual \\
Respeto a autoridades comunales & Inexistencia de autoridades comunales \\
Actores colectivos & Actores individuales \\
Valores de grupo & Valores individuales \\
Conocimiento intergeneracional & Conocimiento institucional \\
Conocimiento oral y colectivo & Conocimiento con soporte escrito \\
Oralidad & Lectoescritura \\
Tradición popular oral & Formas escritas y cultas \\
Vida cotidiana & Sistema educativo oficial \\
Uso de lengua indígena & Uso de la lengua nacional \\
No es necesaria la alfabetización & Necesidad de alfabetización \\
\hline
\end{tabular}

Para la memoria tradicional la oralidad es importante en la reproducción de la memoria y de la opinión cotidiana. Es un instrumento horizontal al que todos tienen acceso; además, supone acercamiento e intimidad, relación directa, contacto, persuasión. Al transmitirse, la oralidad se conserva, a diferencia de lo pictográfico y lo escrito, que puede ser destruido. Los componentes de la oralidad son lo cotidiano, la memoria, las ideas; que integran "la clandestinidad profunda de lo propio” (Martínez, 2003: 61). Es el modelo de reproducción de la vida y la fuente primaria de comunicación, que riñe frente a lo escrito. Sin embargo, debido a que la lectoescritura es la base de la relación con el mundo de la memoria cívica y que mediante ella se negocia parte de la justicia cotidiana, se debe implementar porque es un auxiliar en la reproducción del pensamiento y en el establecimiento de relaciones sociales. Es decir, la lectoescritura es un instrumento comunicativo que tiende 
puentes que van a permitir salvar los ríos de las diferencias culturales. Cabe apuntar que, en el caso de las lenguas indígenas, elaborar un lenguaje escrito es complejo porque se carece de cultura lectoescritural, aunque en México durante los últimos años ha habido avances significativos al respecto. ${ }^{4}$

Un sistema oral se articula con profundidad histórica y tiene un vínculo fundamental con la lengua y con sus modificaciones, así como con procesos simbólicos. Franco llama a dicho sistema "enciclopedia tribal", la cual reproduce saberes sociales: los estructura, los ejecuta, los conserva y los transmite (Franco, 1997: 61-65). El sistema oral y, por tanto la lengua, generan comunidades forjando solidaridades particulares (Anderson, 1997).

Existe otro elemento como medio de expresión y comunicación: la imagen. Lo que se ve nadie lo cuestiona, pues demuestra lo certero. La imagen ilustra, identifica, dice y comunica, reproduce lo que se es: se erige en un discurso preelaborado que se sujeta a diversas interpretaciones.

Si bien oralidad e imagen forman parte de la conducta comunitaria, en el caso de la memoria cívica puede afirmarse que junto al uso de imágenes predomina la lengua escrita -nacional, por supuesto-.

Entonces, en el nivel de la oralidad de la memoria tradicional se perfila la lectoescritura de la memoria cívica. La capacidad de imprimir textos permite su reproducción masificada lo que, al ser utilizado exclusivamente por un Estado educador representa, al mismo tiempo, la posibilidad de estandarización y unificación social. Para la memoria cívica la educación escolarizada es un elemento de vehiculación de la lengua y la cultura, que va a iniciar a los miembros de la sociedad en el conocimiento de los valores y criterios de la vida pública.

\subsection{Elementos de la memoria tradicional}

Existe un concepto, elaborado por intelectuales indígenas, que tiene relación directa con el de memoria cultural: comunalidad. Esta propuesta se sustenta en el pensamiento y la acción de la vida comunitaria, es la suma de sus valores y de las individualidades (Martínez, 2003). Tiene su sustento en el modo de vida y en

\footnotetext{
${ }^{4}$ Los primeros esfuerzos sistemáticos por contar con libros bilingües empezaron a darse en los años cuarenta, cuando la SEP imprimió cartillas bilingües para castellanizar a la población indígena. Actualmente, la Comisión Nacional de los Libros de Texto Gratuitos edita 189 libros en 55 variantes de 33 lenguas indígenas.
} 
la organización del tejido social de la comunidad (Maldonado, 2005). En este caso se trata de una comunidad geométrica y consensual, no aritmética o acumulativa como la occidental, que masifica al individuo pero a la vez lo separa; es decir, es más que la suma de individuos y se define por el territorio, el consenso en la toma de decisiones, el ejercicio de la autoridad como un servicio gratuito, el trabajo colectivo y los ritos y ceremonias (Díaz, 2001). El ejercicio cotidiano de la comunalidad lo convierte en un modelo comunicado generacionalmente.

A partir de dicho concepto, estos autores plantean la existencia de cuatro elementos fundamentales y distintivos de la comunalidad: el poder, el territorio, el trabajo y la fiesta.

\subsubsection{Poder comunal}

En la organización política comunitaria, la autoridad -que no el poder- se sustenta en la asamblea general de ciudadanos o asamblea comunitaria y en el sistema de cargos (sistema de puestos de gobierno comunitario), que tiene una estructura definida. Bautista $^{5}$ y Alcántara ${ }^{6}$ señalan que este derecho indígena -colectivose contrapone al derecho positivo -individual- y también implica la autodeterminación de los pueblos.

Las discusiones de la asamblea pueden desarrollarse en la lengua originaria, que se caracteriza por su oralidad. El debate que se realiza es para discutir y convenir las características de las personas a elegir en los diferentes cargos, los cuales se van nombrando de manera jerárquica a través de ternas, en un ambiente que se pretende sea armónico a fin de que haya acuerdos y adecuaciones en aras de la concordia social. Es decir, el mecanismo se dirige a articular, no a confrontar. Los cargos tienen la obligación interna -no constitucional- de servir gratuitamente a la comunidad, durante varios años.

Se considera que el poder que resulta de la asamblea comunitaria carece de la intervención de ideologías políticas partidistas, no presenta discordias por el dinero ni enriquecimiento ilícito. Además, la estructura de funcionamiento de la autoridad se va

${ }^{5}$ Melitón Bautista López es originario del pueblo zapoteco de San Juan Tabaá, Villa Alta, en la Sierra Norte de Oaxaca; se desempeñó como presidente municipal en 1994 y fue presidente de la Organización de Autoridades Municipales del Sector Zoogocho (1994, 1997, 1998).

${ }^{6}$ Honorio Alcántara Núñez nació en Totontepec mixe, en la Sierra Norte de Oaxaca; en 1986 cumplió con el cargo de presidente municipal y en 2002 con el de alcalde único constitucional. 
adecuando y adaptando a las necesidades de la comunidad (Alcántara, 2004). Es decir, el derecho consuetudinario es un derecho evolutivo; a pesar de que el principio de igualdad jurídica del siglo XIX privó a los grupos étnicos de su derecho consuetudinario (Cordero, 1997).

Sin embargo, hay voces que afirman que esta estructura jurídica comunitaria ha sido violentada por diferentes fenómenos que afectan los derechos colectivos, como "la introducción de personas ajenas a la comunidad y por la educación oficial, la cual introduce todo lo de afuera hacia adentro, no retomando los valores culturales y los derechos de los pueblos y comunidades indígenas" (Bautista, 2004: 42). Otros elementos que han incidido en la ruptura del poder de la asamblea y en la modificación del sistema de cargos son el caciquismo, los partidos políticos, la migración y el protestantismo (Cordero, 1997).

Existe una serie de ceremonias y rituales asociados al ejercicio del poder, de carácter propiciatorio, de purificación y de agradecimiento que se celebran en lugares considerados sagrados, con un valor altamente simbólico. Estas prácticas han tenido cambios ocasionados por la celebración de las fiestas nacionales, por la politización de rituales de las autoridades e incluso por la separación de la organización civil de la religiosa. En la segunda mitad del siglo xx las transformaciones fueron provocadas por la introducción de partidos políticos, el desarrollo de la economía y por las comunicaciones. A ello se debe que en algunos pueblos el sistema se haya modificado, como la paulatina desaparición del Consejo de Ancianos, la pérdida de las Mayordomías o la disminución de la importancia del cargo de Alcalde (Cordero, 1997). Curiosamente, en la conservación de los rasgos del sistema tradicional tuvo que ver la no satisfacción, por el Estado nacional, de las necesidades interiores de la comunidad, la falta de comunicaciones, el monolingüismo y la lejanía de algunos sitios respecto de los centros urbanos.

En términos legales este poder comunal lo reconoce el Código de Instituciones Políticas y Procedimientos Electorales de Oaxaca (1995) como usos y costumbres, aunque Martínez propone utilizar el concepto de comunalicracia como modelo político, concepto que alude a la calidad, la competencia y el prestigio para ejercer autoridad con base en el poder que emana de la asamblea general, puesto que lo asambleario garantiza la relación directa entre poder ejecutivo y población. En este sentido el cargo de representación adquiere un factor de calidad, distinto 
al carácter de cantidad que se reproduce por medio de la democracia representativa nacional. Comunalicracia implica la disolución de cualquier posible sociedad política en la sociedad civil. La representación es temporal y obligatoria, por lo mismo no permite la especialización ni lo oficioso que conduce a la hegemonía del poder y su concentración en pocas personas (Martínez, 2003: 50).

\subsubsection{Territorio comunal}

El territorio está dado como comunal e indivisible a la comunidad, representado por las autoridades agrarias; también es comunal porque es un territorio sagrado (es decir, en él se dan manifestaciones y expresiones de lo sobrenatural). El territorio comunal implica la comunidad territorial. Más aún, se considera que el territorio es la madre tierra del pueblo o la comunidad que se habita, ya que es el espacio de reproducción de los mitos (cosmovisión), donde se vive en comunalidad, donde todo se realiza en común. El territorio se comunaliza para sobrevivir.

\subsubsection{Trabajo comunal}

Hay dos formas de trabajo comunal: el tequio y la ayuda mutua. El tequio es el trabajo gratuito, constante y obligatorio de todos los ciudadanos que participan en obras de beneficio comunitario. Puede ser de barrio, comunitario e intercomunitario. Es tal su arraigo que el gobierno ha recurrido a este tipo de trabajo para optimizar el uso de recursos destinados a obra pública: en el sexenio del gobernador Heladio Ramírez López (1986-1992) hubo un programa oficial llamado Lluvia, tequio y alimentos, semejante en su operación al Programa Nacional de Solidaridad del gobierno federal (1988-1994).

Si el trabajo comunal es para beneficio familiar y de apoyo entre familias, se llama ayuda mutua y es un mecanismo de igualación simbólica; dicha ayuda es solidaria porque una relación pagada carece de reciprocidad.

En la vida comunal el cumplimiento de obligaciones se liga al otorgamiento de derechos: quien se aleja de la comunalidad se aísla de la comunidad. Si alguien evade sus obligaciones significa que hay desigualdad, por eso no hay (o no debe haber) privilegios. 


\subsubsection{Fiesta comunal}

La fiesta se organiza en un contexto de ayuda mutua y de reciprocidad, no es selectiva; existen varios tipos de fiesta: comunitaria, familiar y de convivencia ritual. En la fiesta se manifiesta la identidad (a nivel personal, comunitario, regional y étnico) a través de elementos como la música, la danza, la indumentaria, los alimentos y las bebidas, la lengua y la convivencia comunal. La comunidad festiva, al ser un espacio de armonía y de resignificación de lo tradicional, refuerza la pertenencia comunitaria.

\subsection{Componentes de la memoria cívica}

En el caso del Estado nacional, estos cuatro elementos (el poder, el territorio, el trabajo y la fiesta) también están presentes y son parte importante de la memoria cívica, con características propias entre las que cabe destacar su reproducción institucional, sobre todo a través de sistemas escolarizados. El soporte de estos elementos se encuentra en la legislación nacional y en las disposiciones legales que cada una de las entidades federativas tiene.

\subsubsection{El poder}

En términos constitucionales, México es una república representativa, democrática y federal, integrada por estados federados, libres y soberanos (art. 40). La base de la organización política es el sistema representativo -mediante elecciones libres y periódicas- con partidos políticos que deben promover la participación del pueblo en la vida democrática y representativa, haciendo posible el acceso de los ciudadanos al poder público (art. 41). El supremo poder de la federación se divide, para su ejercicio, en ejecutivo, legislativo y judicial (art. 49); sin embargo, debido a que la soberanía reside esencialmente en el pueblo, éste tiene el derecho de alterar o modificar, en todo tiempo, la forma de gobierno (art. 39).

La Constitución señala que son ciudadanos de la República los mexicanos que hayan cumplido 18 años de edad y tengan un modo honesto de vivir (art. 34); con derechos (votar y ser votado para cargos de elección popular, libertad de asociación política, participar en el ejército para defender la República) y obligaciones (inscribirse en el registro nacional de ciudadanos, alistarse en la Guardia Nacional, votar en las elecciones, desempeñar los 
cargos concejiles del municipio donde resida). El capítulo I de la Constitución está compuesto por 29 artículos que son las garantías individuales, entre las que se encuentran: la prohibición de la discriminación; el reconocimiento de México como nación pluricultural; el derecho a la educación que será laica, democrática y nacional; el derecho a las necesidades básicas y al trabajo; la libertad de expresión, de asociación y de tránsito; la libertad de culto y el derecho a la propiedad privada.

El conocimiento de estos elementos del poder, que vinculo con lo que llamo memoria cívica, se transmite fundamentalmente a través del sistema de educación básica.

\subsubsection{El trabajo}

Los mexicanos tienen derecho al trabajo, el cual se debe realizar con su consentimiento y mediante una retribución justa (art. 5); esta actividad es resultado del esfuerzo individual en el que el sujeto participa de acuerdo a su capacidad y competencia, generalmente mediante la demostración de sus aptitudes y capacidades. Legalmente, las relaciones laborales están reguladas por la Ley Federal de Trabajo y por una serie de disposiciones y normas que las reglamentan, estipulando los mecanismos mediante los cuales se estructuran dichas relaciones en cuanto a jornada laboral, salarios y prestaciones.

En este caso se habla de trabajo individual, concepción diferente a la del trabajo dentro de la memoria tradicional, donde puede ser individual y colectivo.

\subsubsection{La fiesta}

Las fiestas que habitualmente convocan a la generalidad de los mexicanos son las de carácter cívico, cuya observación en las escuelas es obligatoria por lo que involucran, además de a los estudiantes, a la familia. Para el cumplimiento de estos festejos existe un calendario oficial de estricta observancia, mismo que ha sido un pilar fundamental en la construcción del imaginario nacional y en la enseñanza de la historia. Así, las fiestas principales tienen que ver con momentos considerados primordiales en la historia (independencia, revolución) y con el natalicio de los héroes nacionales. Es común que estos festejos tengan una fuerte carga política descontextualizándose, la mayoría de las veces, de su significado histórico. 


\subsubsection{El territorio}

Los límites del territorio nacional se establecen a partir de tratados y acuerdos de carácter internacional, por lo que la frontera es resultado de acuerdos binacionales que pueden obedecer a accidentes geográficos o a trazos definidos de manera conjunta y hasta cierto punto de forma arbitraria. La Constitución mexicana señala que el territorio está comprendido por las partes que componen la federación (es decir, los estados), además del espacio sobre dicho territorio, el mar territorial y las islas (arts. 42 y 43).

\section{A manera de conclusión}

Se pueden señalar algunos ejemplos de sincretismo, de concordancia entre ambos tipos de memoria en diferentes momentos de la vida pública de los pueblos. En el caso de Oaxaca, aproximadamente $73 \%$ de los municipios (418 de 570) elige a sus autoridades mediante el sistema conocido como usos y costumbres, esto es, a través de asambleas comunitarias en las que se nombra el poder comunal descrito anteriormente. En muchos de estos municipios, como en los pueblos mixes de la Sierra Norte, el cambio de autoridades se realiza el 31 de diciembre por la noche; con motivo de este cambio se realizan ritos propiciatorios en cuevas y caminos a la par que se llevan a cabo ceremonias cívicas con honores a la bandera nacional.

Otro ejemplo que se puede citar es el que tiene que ver con la reciente creación de un municipio autónomo en la región trique, al poniente de Oaxaca. El 20 de enero de 2007 se formalizó la creación del municipio autónomo de San Juan Copala, en cuya ceremonia se pudo apreciar a mujeres triques vestidas a la usanza indígena y portando la bandera nacional, o a niñas con sus huipiles tradicionales portando los tambores de la banda de guerra, cuyos bordes estaban decorados con los colores de la bandera nacional. Más aún, pues al margen de estos dos elementos que pueden caer en el terreno de lo simbólico, tres de los cinco puntos de la declaración del municipio autónomo señalan:

Segundo: Las autoridades del municipio autónomo de San Juan Copala son aquéllas que las comunidades y barrios que integran el municipio autónomo han elegido libremente, a las cuales ha dado posesión el Consejo de Ancianos. Estas autoridades podrán ser destituidas en cualquier momento si atentan contra la voluntad del pueblo o se subordinan a las políticas del gobierno. 
Cuarto: Las autoridades del municipio autónomo de San Juan Copala sujetarán sus actos a los usos y costumbres del pueblo trique y, en lo que éstos no prevean forma de conducirse, a las leyes del Estado mexicano. Quinto: Las autoridades del municipio autónomo de San Juan Copala representarán a las comunidades y barrios hacia el exterior del municipio, respetando siempre la voluntad de sus ciudadanos y el respeto de la cultura trique (La Jornada Ojarasca, 2007: 7).

Al principio de este texto apunté que ambos tipos de memoria se hibridan y que la arbitraria separación que he propuesto pretendía diseccionar los elementos que las integran, para una mejor comprensión de cada una de ellas y de ambas a la vez. Pero los dos tipos de memoria se articulan cotidianamente y tienen consecuencias en la vida pública y social. Sin embargo, durante el siglo xx los pueblos indígenas objetivo histórico de civilización resintieron las políticas públicas encaminadas a implantar de manera hegemónica la memoria cívica; sólo a partir de las últimas dos décadas de dicho siglo se replantearon las ideas, los proyectos y las formas de relación entre lo indígena y lo que no lo era, a fin de establecer un diálogo menos vertical y con resultados sociales cuestionables, al menos en Oaxaca.

La dicotomía entre memoria tradicional y memoria cívica no es una cuestión polarizada; las dos no son tan distintas y se mezclan, dando lugar a una esencia que es precisamente el sincretismo y no la diferencia extrema. Lo que hace la identidad de las comunidades es precisamente la alquimia particular que producen, con normas y prácticas de la sociedad nacional y heredadas. Conocer mejor los elementos de la vida cotidiana de dichos pueblos, como su memoria tradicional, contribuirá a que dicho diálogo adquiera un mayor significado en la construcción de una sociedad más justa y digna.

\section{Bibliografía}

AHSEP (Archivo Histórico de la Secretaría de Educación Pública).

Alcántara-Núñez, Honorio (2004), Usos y costumbres. Vivencias $y$ convivencias de un alcalde mixe, Conaculta, México.

Anderson, Benedict (1997), Comunidades imaginadas. Reflexiones sobre el origen y la difusión del nacionalismo, Fondo de Cultura Económica, México. 
Bautista-Cruz, Melitón (2004), Normas de convivencia a través de acuerdos comunitarios, Conaculta-Instituto Oaxaqueño de las Culturas, Oaxaca, México.

Cordero-Avendaño, Carmen (1997), La vara de mando. Costumbre jurídica en la transmisión de poderes, $\mathrm{H}$. Ayuntamiento de Oaxaca de Juárez, Oaxaca, México.

Díaz-Gómez, Floriberto (2001), “Comunidad y comunalidad”, La Jornada Semanal, 11 de marzo, versión electrónica: http:// www.jornada.unam.mx/2001/03/11/sem-comunidad.html.

Franco-Pellotier, Víctor M. (1997), "Simbolismo y oralidad", Alteridades, 13, Universidad Autónoma Metropolitana, México, pp. 61-65.

Gramsci, Antonio (1975), Cuadernos de la cárcel, ERA, México.

La Jornada Hojarasca (2007), suplemento mensual, número 118, febrero.

Halbwachs, Maurice (2004), Los marcos sociales de la memoria, Anthropos, Barcelona, España.

Maldonado, Benjamín (2005), Desde la pertenencia al mundo comunal. Propuestas de investigación y uso de experiencias y saberes comunitarios en el aula indígena intercultural de Oaxaca, Centro de Estudios Ayuuk, Oaxaca, México.

Martínez-Luna, Jaime (2003), Comunalidad y desarrollo, Conaculta-Campo, México.

Pitarch, Pedro (2002), "Un lugar difícil: estereotipos étnicos y juegos de poder en los Altos de Chiapas", en Juan Pedro Viqueira y Mario Humberto Ruz (eds.), Chiapas, los rumbos de otra historia, UNAM-CIESAS, México, pp. 237-250.

Vigotsky, Lev (1996), Pensamiento y lenguaje: teoría del desarrollo cultural de las funciones psíquicas, Quinto Sol, México.

Recibido: 15 de marzo de 2007. Aceptado: 9 de septiembre de 2007. 
Salvador Sigüenza Orozco. Es doctor en historia por la Universidad Complutense de Madrid; actualmente se encuentra adscrito al Centro de Investigaciones y Estudios Superiores en Antropología Social del CIESAS, Unidad Pacífico Sur (antes Oaxaca), y es profesor en la Universidad José Vasconcelos de Oaxaca. Su línea de investigación actual es historia de la educación. Entre sus últimas publicaciones destacan: Héroes y escuelas. La educación en la Sierra Norte de Oaxaca (1927-1972), INAH-IEEPO, Oaxaca, México, 2007; “1972: reforma educativa y valores cívicos en la escuela primaria", Educación 2001, 141, pp. 56-60 (2007); "Oaxaca: educación y cultura en los años cuarenta", Identidades, Revista de Educación y Cultura, $2^{\mathrm{a}}$ época, año IV, 15, Instituto Estatal de Educación Pública de Oaxaca, pp. $49-57$ (2005); "La idea de nacionalidad en los Libros de Texto Gratuitos de México (1959-1972)", Tzintzun, Revista de Estudios Históricos, 41, Universidad Michoacana de San Nicolás de Hidalgo, Morelia, pp. 57-82 (2005); "Maestros, misiones culturales y normales rurales en Oaxaca: los años treinta", Acervos, Boletín de los Archivos y Bibliotecas de Oaxaca, 7(26), México, pp. 25-32 (2004). 